\title{
Cost-effectiveness analysis of repeated self- sampling for HPV testing in primary cervical screening: a randomized study
}

\author{
Riina Aarnio ${ }^{1 *} \mathbb{D}$, Ellinor Östensson ${ }^{2,3}$, Matts Olovsson $^{1}$, Inger Gustavsson ${ }^{4}$ and Ulf Gyllensten ${ }^{4}$
}

\begin{abstract}
Background: Human papillomavirus (HPV) testing is recommended in primary cervical screening to improve cancer prevention. An advantage of HPV testing is that it can be performed on self-samples, which could increase population coverage and result in a more efficient strategy to identify women at risk of developing cervical cancer. Our objective was to assess whether repeated self-sampling for HPV testing is cost-effective in comparison with Pap smear cytology for detection of cervical intraepithelial neoplasia grade 2 or more (CIN2+) in increasing participation rate in primary cervical screening.
\end{abstract}

Methods: A cost-effectiveness analysis (CEA) was performed on data from a previously published randomized clinical study including 36,390 women aged 30-49 years. Participants were randomized either to perform repeated self-sampling of vaginal fluid for HPV testing ( $n=17,997$, HPV self-sampling arm) or to midwife-collected Pap smears for cytological analysis ( $n=18,393$, Pap smear arm).

Results: Self-sampling for HPV testing led to 1633 more screened women and 107 more histologically diagnosed CIN2+ at a lower cost vs. midwife-collected Pap smears ( $€ 229,446$ vs. $€ 782,772$ ).

Conclusions: This study resulted in that repeated self-sampling for HPV testing increased participation and detection of CIN2+ at a lower cost than midwife-collected Pap smears in primary cervical screening. Offering women a home-based self-sampling may therefore be a more cost-effective alternative than clinic-based screening.

Trial registration: Not registered since this trial is a secondary analysis of an earlier published study (Gustavsson et al., British journal of cancer. 118:896-904, 2018).

Keywords: Self-sampling, HPV testing, Primary cervical screening, Cost-effectiveness, CIN2 +, Precancerous lesion, Cervical cancer

\section{Background}

Organized screening with Papanicolaou cytology (Pap smear) has resulted in a major reduction in both the incidence of cervical cancer and related mortality [1]. Nevertheless, about 500 women are diagnosed with cervical cancer, and about 140 women die of it every

\footnotetext{
* Correspondence: riina.aarnio@kbh.uu.se

'Department of Women's and Children's Health, Uppsala University, 75185 Uppsala, Sweden

Full list of author information is available at the end of the article
}

year in Sweden [2]. Persistent infection with oncogenic high-risk types of human papillomavirus (HPV) is a prerequisite for the development of cervical cancer [3], although most HPV infections clear spontaneously, with no increased risk for cervical cancer. HPV testing has greater sensitivity in revealing histological cervical intraepithelial neoplasia grade 2 or more $(\mathrm{CIN} 2+)$ than cytology $[4,5]$, and primary cervical screening by means of HPV testing is recommended in Europe [6, 7]. Because of the lower 
specificity of HPV testing, cytological testing of HPVpositive samples at primary screening (cytology triage) is recommended. This presents a challenge in how to manage women that are HPV-positive but cytologynegative, since they have an elevated risk of CIN2+ [8]. In a previous study, we proposed that repeated HPV testing to identify persistent infections can be used as an alternative to cytology triage [9]. Among women with an HPV-positive screening result, 70\% had an HPV infection when retested 4-6 months later, and repeated HPV testing is estimated to result in similar overall specificity as with cytology based screening.

A low population coverage of screening has been identified as an important risk factor of incident cervical cancer [10]. Previous studies have revealed increased response rates among non-responders by offering selfsampling kits for HPV testing, vs. other options [11-16]. In a large meta-analysis self-sampling for primary cervical screening was recommended, but only when using PCR-based HPV tests [17].

An important criterion for a screening program is the cost-effectiveness (https://www.socialstyrelsen.se/ globalassets/sharepoint-dokument/artikelkatalog/nationella-screeningprogram/2019-4-12.pdf), and healtheconomic evaluations are therefore needed before deciding on implementation of new screening tests or strategies. The first aim of this study was to compare the cost-effectiveness of repeated self-sampling for HPV testing with midwife-collected Pap smear cytology based on data from a recent randomized study on primary cervical screening [18]. The second aim was to estimate the cost of treatment and follow-up of histological CIN2 + .

\section{Methods}

\section{Study design}

This study is a secondary analysis based on clinical and cost data from a previously published randomized study [18]. During 2013-2015 a total of 36,390 women aged 30-49 years planned for regular screening invitation in Uppsala, Sweden, were randomized into two arms; a) repeated self-sampling of vaginal fluid for HPV testing ( $n=17,997$, HPV self-sampling arm) or to midwifecollected Pap smear for cytological analysis $(n=18,393$, Pap smear arm) [18]. The study flowchart is shown in Fig. 1 and number of women included are shown in Fig. 2. Women with previous hysterectomy or current pregnancy were recommended in the invitation letter not to participate in the study. Some of these women nevertheless performed self-sampling and women with previous hysterectomy were excluded after second selfsampling, while the pregnant women were included in this study. A cost-effective analysis (CEA) was performed comparing the alternative screening strategies, based on quantification, effectiveness and cost data. Additional treatment and follow-up data were collected from patient files.

\section{HPV self-sampling arm}

Women were sent an invitation with a self-sampling kit for vaginal fluid, including a sampling brush, an FTA card, a step-by-step guide on how to perform the sampling and a pre-addressed and postage-paid return envelope. Women performed the self-sampling and sent the FTA card to the Department of Immunology, Genetics and Pathology at Uppsala University (HPV laboratory) for HPV testing. Women with a positive HPV test result were sent a second self-sampling kit for repeat sampling

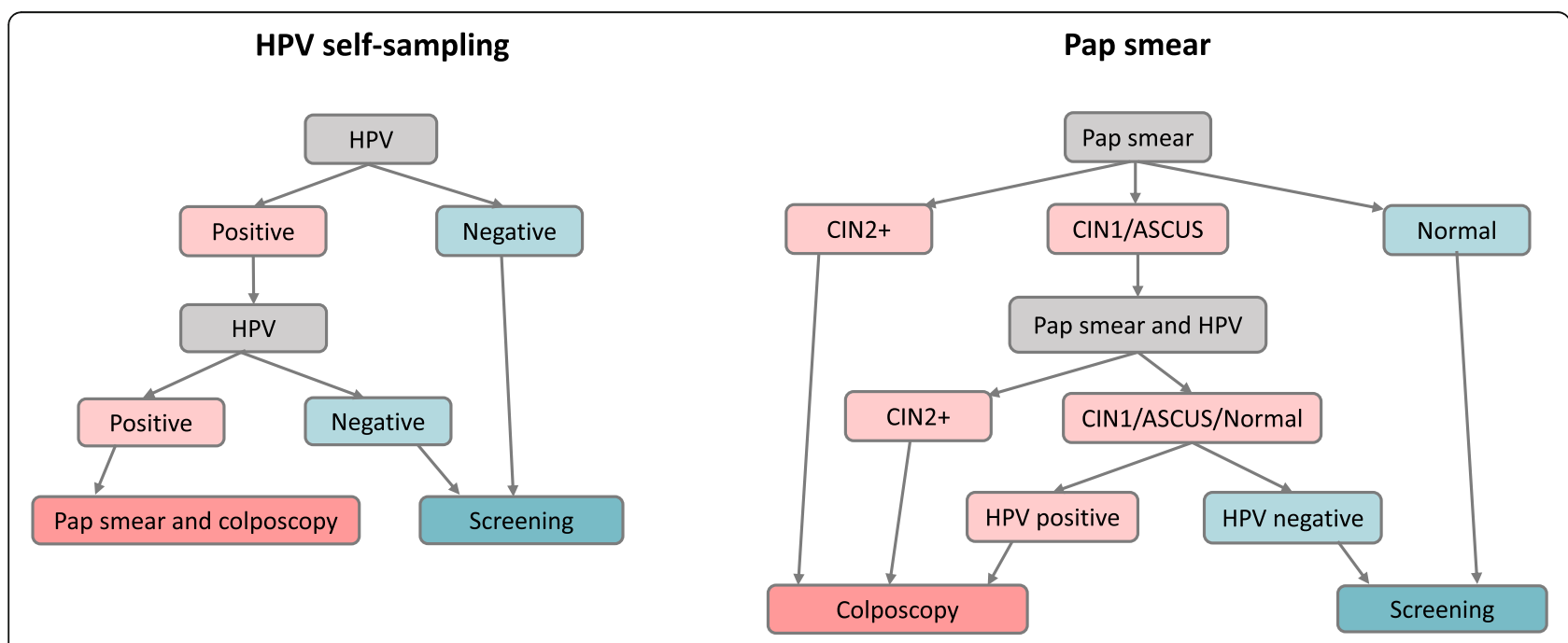

Fig. 1 Study protocol flowchart in the HPV self-sampling and Pap smear arms 


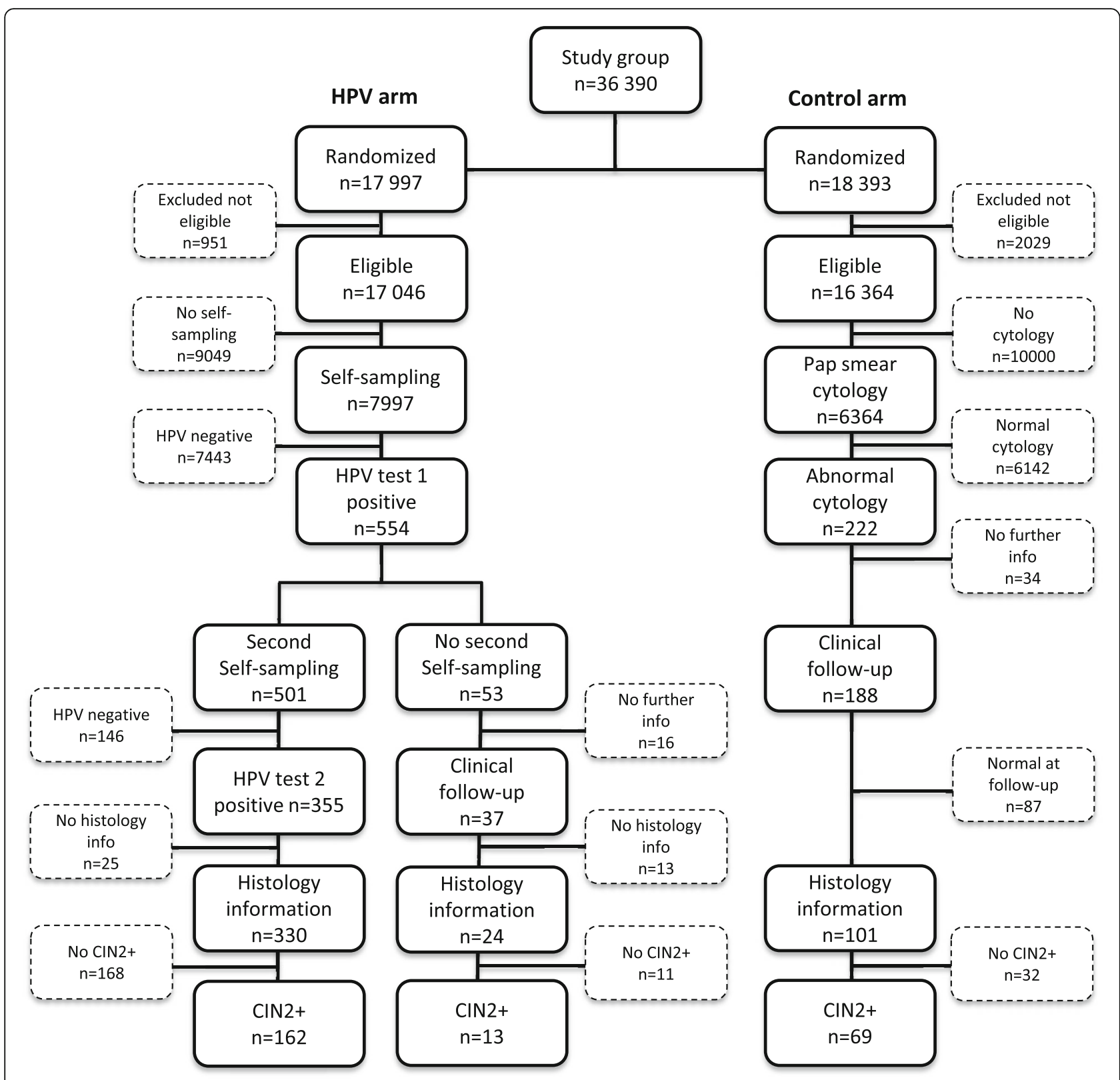

Fig. 2 Study flowchart with number of women at different steps in the HPV self-sampling and Pap smear arms

after 3-6 months. Participation in primary screening was classified as complete when having an analyzed first negative HPV test, or in the case of a first positive HPV test, after repeated HPV testing. Women with two consecutive HPV-positive self-samples were referred to colposcopy. All HPV-negative women were referred back to screening (Fig. 1). Details on sampling material, processing and HPV analysis are described in the published study [18].

\section{Pap smear arm}

Women were sent an invitation to schedule an appointment at the local midwife clinic, where Pap smear sampling for cytology was performed. Participation in primary screening was classified complete when having an analyzed Pap smear. Women with CIN2+ in cytology were referred to colposcopy. Women with low-grade cervical intraepithelial neoplasia (CIN1) or atypical squamous cells of unknown significance (ASCUS) in cytology were scheduled for repeated Pap smear and HPV test by a midwife after 3 months. HPV-positive women and women with CIN2+ in cytology were referred to colposcopy, while HPV-negative women without CIN2+ in cytology were referred back to screening (Fig. 1). Cytology and histology were performed at the Department of 
Pathology and Cytology, Uppsala University Hospital. Pap smears and histological diagnoses were classified according to CIN terminology. The highest histological grade found in each patient was used for interpretation.

\section{Colposcopy}

Women with repeated HPV-positive results, ASCUS/ CIN1 cytology and HPV-positive result or CIN2+ cytology were followed up by colposcopy. Here, the squamocolumnar junction and transformation zone were identified, $5 \%$ acetic acid and iodine solution were applicated and after visual evaluation, all lesions were biopsied. A Pap smear was collected on all HPV-positive women in the self-sample arm. In cases of transformation zone 3 (TZ3) a sample was also collected for endocervical cytology. Mainly one expert colposcopist performed colposcopies among women in the HPV selfsampling arm, while different colposcopists performed colposcopies in the Pap smear arm.

\section{Treatment of precancerous lesions and cancers}

Women with histological CIN2+ were treated according to current clinical recommendations. In the Pap smear arm, about one fifth of the women with CIN were treated at a regional hospital (Enköping lasarett, Enköping). The rest of the women with CIN and women with cancer were treated at the Department of Gynecology and Obstetrics, Uppsala University Hospital. Precancerous lesions and micro-invasive cancers were treated by using the loop electrosurgical excision procedure (LEEP), most of them under local anesthesia but some under general anesthesia. Treated women were invited for a follow-up appointment ('test of cure') with a midwife or a gynecologist in 4-6 months. At this appointment, the midwives collected a Pap smear and a sample for HPV testing, and in addition to them, the gynecologist also carried out colposcopy. The cancer cases were discussed at a multidisciplinary meeting after requisite radiological investigation, usually chest and abdominal CT scans and a pelvic MR scan. Surgical treatment consisted of either simple or radical hysterectomy or trachelectomy. Radical surgery included excision of the upper vagina and parametria with bilateral pelvic lymphadenectomy beyond removal of the uterus (hysterectomy) or the cervix (trachelectomy). Surgery was performed either by laparotomy or in most cases by means of minimally invasive techniques, such as laparoscopy or robotic-assisted laparoscopic surgery.

\section{Outcome data}

Clinical data (at the time of screening invitation and the cytological and histological test results at clinical followup) were retrieved from a database at the Department of Pathology and Cytology, Uppsala University Hospital.
All events from invitation until diagnosis were noted for each patient in both study arms. The treatment records, including further preoperative assessment and follow-up after treatment in cases of $\mathrm{CIN} 2+$, were manually checked in the patient files until 31 December 2018. All events were included after LEEP until the 'test of cure' was accepted (HPV-negative and Pap smear cytology < CIN2), or after surgical treatment of cancer, until the first postoperative visit. Possible treatments and followup in cases of CIN1 were not included in this analysis.

\section{Cost-effectiveness analysis (CEA) and cost estimation}

A CEA was performed using healthcare provider perspective [19]. The unit costs for each screening event were retrieved from the HPV laboratory and Uppsala region financial records. Direct medical costs of inpatient and outpatient healthcare were retrieved from the financial records at Uppsala University Hospital. When needed costs were adjusted for inflation by using the consumer price index (CPI) [20] and converted to 2019 Euros (mean annual exchange rate, $€ 1=10.5912$ SEK). A cost per screened woman was calculated in each study arm according to the study protocol. Screening strategies (HPV self-sampling vs. Pap smear) were ranked from the lowest to the most costly. Incremental cost-effectiveness ratios (ICERs) per extra screened women were calculated by dividing the cost difference (cost) with the difference in number of screened women (effect) between the two screening arms in the randomized study. At clinical follow-up, also the ICERs per extra detected woman with CIN2+ were calculated. If a screening arm was more costly and less effective than the comparative one, it was defined as strongly dominated. A sensitivity analysis was performed to account for the uncertainty of screen participation and trends in direct medical costs. Moreover, using the cost data we estimated the cost of treatment and follow-up of histological CIN2 + .

\section{Results \\ Cost-effectiveness analysis on primary screening including clinical follow-up}

The participation rate in cervical screening was significantly higher in the HPV self-sampling arm than in the Pap smear arm (47\% vs. $39 \%, P<0.001)$. In the HPV self-sampling arm, 7997 women returned a self-sample for HPV testing, of which 7443 (93\%) were HPVnegative and considered as completely screened (Table 1). A second self-sampling kit was sent to 554 HPV-positive women and 501 (90.4\%) women returned a sample and considered completely screened. In the second HPV test, 355 women were positive and were referred to colposcopy. In total, 175 cases of histological CIN2+ were identified in the HPV self-sampling arm. In the Pap smear arm, 6364 women visited a midwife for a 
Table 1 Resources required per screened woman by intervention arm, with associated costs in 2019 (€ $1=10.5912$ SEK)

\begin{tabular}{|c|c|c|c|c|c|}
\hline & \multirow[b]{2}{*}{ Unit Cost $(€)$} & \multirow{2}{*}{$\begin{array}{l}\text { Pap smear }(n=18,393) \\
\text { Units }\end{array}$} & \multicolumn{3}{|c|}{ HPV self-sampling $(n=17,997)$} \\
\hline & & & Costs $(€)$ & Units & Costs $(€)$ \\
\hline \multicolumn{6}{|l|}{ Primary screening } \\
\hline Pap smear cytological analysis & 25 & 6364 & 159,100 & & \\
\hline Midwife appointment for sampling & 98 & 6364 & 623,672 & & \\
\hline First HPV self-sampling kit, return and HPV test (including postal fees) & 27 & & & 7997 & 215,919 \\
\hline 2nd HPV self-sampling kit, return and HPV test (including postal fees) & 27 & & & 501 & 13,527 \\
\hline Total cost of primary screening & & & 782,772 & & 229,446 \\
\hline Screened women & & 6364 & & 7997 & \\
\hline Abnormal cytology in need of clinical follow-up & & 222 & & & \\
\hline 2nd HPV-positive in need of clinical follow-up & & & & 355 & \\
\hline Incremental effect (screened women) & & & 1633 & & \\
\hline Cost per screened woman & & & 123 & & 29 \\
\hline ICER per extra screened woman & & & -339 & & \\
\hline \multicolumn{6}{|l|}{ Clinical follow-up } \\
\hline Pap smear cytological analysis & 25 & 238 & 5950 & 428 & 10,700 \\
\hline HPV analysis* & 38 & 173 & 6574 & 46 & 1748 \\
\hline Midwife appointment for sampling & 98 & 166 & 16,268 & 4 & 392 \\
\hline Colposcopy appointment & 470 & 114 & 53,580 & 384 & 180,480 \\
\hline Biopsy histological analysis & 147 & 114 & 16,758 & 367 & 53,949 \\
\hline Total cost of clinical follow-up & & & 99,130 & & 247,269 \\
\hline Total cost of primary screening + clinical follow-up & & & 881,902 & & 476,715 \\
\hline $\mathrm{CIN} 2+$ & & 68 & & 175 & \\
\hline Incremental effect (CIN2+) & & & 107 & & \\
\hline Cost per woman with $\mathrm{CIN} 2+$ & & & 12,969 & & 2724 \\
\hline ICER per extra detected woman with CIN2+ & & & -3787 & & \\
\hline
\end{tabular}

Sensitivity analysis HPV vs. Pap smear (ICER per extra detected woman with CIN2+)

\section{Efficacy parameters}

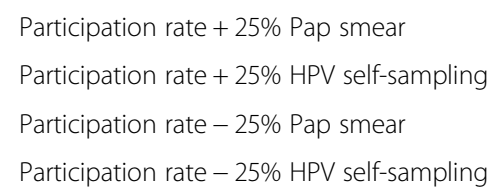

$\begin{array}{lll}7955 & -5451 & \\ & -3124 & 9996 \\ 4773 & -111 & \\ & -4129 & 5998\end{array}$

\section{Screening cost variation}

$\begin{array}{lll}\text { HPV self-sampling }+25 \% & 34 & -3092 \\ \text { HPV self-sampling }-25 \% & 20 & -4160 \\ \text { Pap smear cytological analysis }+25 \% & 32 & -3990 \\ \text { Pap smear cytological analysis }-25 \% & 19 & -3262 \\ \text { Midwife appointment }+25 \% & 122 & -5076 \\ \text { Midwife appointment }-25 \% & 73 & -2176 \\ \text { Midwife appointment }-50 \% & 49 & -727\end{array}$

*Total cost of HPV test including HPV kit and analysis performed at the HPV laboratory, Uppsala University and transfer of the results to the database at the Department of Cytology and Pathology, Uppsala University Hospital

Pap smear and considered completely screened. Among these 6142 (97\%) had normal cytology, whereas 222 women had abnormal Pap smear results and where referred to follow-up. In total, 68 women with histological
CIN2+ were identified in the Pap smear arm (Table 1). All ten cancer cases in the HPV self-sampling arm had FIGO stages 1A1-1B1 without cancer recurrence in May 2020. Of the five cancer cases in the Pap smear arm four 
had FIGO stages 1A1-1B1 without cancer recurrence in May 2020, while one woman with FIGO stage 1B2 had died because of her cervical cancer. Thus, there seems to be no increase in risk for more advanced cancer due to the time span between first and second HPV test.

The total cost of primary screening was higher for the Pap smear arm than for the HPV self-sampling arm ( $€$ 782,772 vs. $€ 229,446$ ), and the Pap smear arm was thus strongly dominated (Table 1). The HPV self-sampling arm also resulted in detection of more cases of CIN2+ at a lower cost in comparison with Pap smear arm and is a cost-saving alternative (clinical follow-up, Table 1). Sensitivity analysis for participation rate, screening test cost (Pap smear analysis and HPV test analysis), selfsampling kit cost and midwife appointment cost did not affect the results (Table 1).

\section{Cost estimation of treatment of CIN2+ including follow- up}

In the Pap smear arm, 68 women had CIN2+ and in the HPV self-sampling arm, 175 women had CIN2+ (Table 2). The total cost of treatment of histological CIN2+, was $€ 444,125$ (192 treatments) in the HPV selfsampling arm and $€ 235,211$ (70 treatments) in the Pap smear arm. Cost per treated woman was $45 \%$ higher in the Pap smear arm $(€ 3675)$ than in the HPV selfsampling arm (€ 2538) (Table 2).

\section{Discussion}

This study demonstrated that repeated self-sampling for HPV testing at home was more effective in increasing participation and detecting CIN2+ and less costly than midwife-collected Pap smear cytology in primary cervical screening. Our results concerning the cost of cervical screening based on self-sampling for HPV testing are in line with those of previous studies modeling the costeffectiveness of HPV testing in primary cervical screening. In a study from Canada it was concluded that using HPV testing both in primary screening or as triage of equivocal Pap smear results was more effective and costeffective relative to cytology [21]. In a study from the Netherlands it was predicted that replacing cytology in primary screening by way of HPV testing and cytology triage would increase the total cost, but this could be compensated for by extended screening intervals [22]. A study from Australia, including a vaccinated population, showed that primary HPV testing with partial genotyping of HPV16/18 every 5 years was a more effective and less costly strategy than cytology screening every 2 years [23]. In triage of cytological ASCUS or LSIL, genotyping for HPV16/18 has shown to be the most cost-effective strategy [24].

Self-sampling for HPV testing is one of the most effective (in improving participation) and cost-effective interventions as regards non-responders, and has been evaluated in several European populations [25-29]. Similar to our study results, previous intervention studies for sensitivity analysis on participation rate, health care costs, Pap smears and self-sampling kits have not affected the results. Our published randomized study was the first to demonstrate an increase in participation in primary screening with vaginal self-sampling using PCR-based HPV test, as compared with midwifecollected Pap smear cytology [18]. In the present study we provide a cost-effectiveness analysis of the randomized trial. The total cost per woman participating in primary screening was 4.2 times higher in the Pap smear arm than in the HPV self-sampling arm.

The estimation of treatment costs showed that the cost per treated woman was $45 \%$ higher in the Pap smear arm, since more women with cervical intraepithelial neoplasia grade 2 (CIN2) were detected by HPV selfsampling than by Pap smears. This, together with somewhat different treatment policies in different hospitals, resulted in more excisions under local anesthesia and proportionally fewer hysterectomies in the HPV selfsampling arm than in the Pap smear arm, resulting in a lower cost per treated woman. During the study period about $50 \%$ of all women visited a gynecologist for colposcopy as follow-up after treatment ('test of cure'). According to present guidelines the recommended 'test of cure' is cytology and HPV testing based on a sample collected by a midwife. As previous studies have showed that only up to about $30 \%$ of patients are HPV-positive 6 months after treatment [30-32], only these might need a colposcopy, resulting in lower costs for the HPV selfsampling arm.

One strength of our CEA is that we retrospectively collected healthcare events for all included patients in a randomized trial. We then applied the direct medical costs reported from the financial records, together with costs of self-sampling kits and postal fees to each individual patient. The data therefore provide reliable estimates of costs.

This study included all direct medical costs, but not all direct costs (e.g. transportation costs to and from the clinic, parking fees or childcare costs) and indirect costs (i.e. those corresponding to the 'time off work' needed for scheduling and conducting the screening appointment) related to clinician-collected samples. These costs can be substantial [33] and including them would result in a more comprehensive estimate of the actual differences in costs between alternative strategies for primary cervical screening. 'Time off work' can also represent a barrier to attending clinic-based screening, and avoiding such barriers thus might lead to higher population coverage [33]. In our previous CEA study we compared self-sampling for HPV testing with Pap smear cytology 
Table 2 Resources required for treatment including further preoperative assessment and follow-up of CIN2+ among women by intervention group with associated costs in 2019 ( $€ 1=10.5912$ SEK)

\begin{tabular}{|c|c|c|c|c|c|}
\hline & \multirow[b]{2}{*}{ Unit Cost $(€)$} & \multirow{2}{*}{$\begin{array}{l}\text { Pap smear } \\
\text { Units }\end{array}$} & \multicolumn{3}{|c|}{ HPV self-sampling } \\
\hline & & & Costs $(€)$ & Units & Costs $(€)$ \\
\hline CIN2+ histological analysis & 775 & 68 & 52,700 & 175 & 135,625 \\
\hline Pregnant & 0 & 10 & 0 & 11 & 0 \\
\hline \multicolumn{6}{|l|}{ Treatment } \\
\hline Excision (local anesthesia) & 753 & 40 & 30,120 & 151 & 113,703 \\
\hline Excision (general anesthesia) & 1767 & 23 & 40,641 & 33 & 58,311 \\
\hline Hysterectomy (mini-invasive) & 5825 & 3 & 17,475 & 5 & 29,125 \\
\hline Hysterectomy/trachelectomy (radical) & 11,973 & 4 & 47,893 & 3 & 35,919 \\
\hline Total number of treatments & & 70 & & 192 & \\
\hline Inpatient care (mean) & 968 & 24 & 23,232 & 39 & 37,752 \\
\hline \multicolumn{6}{|l|}{ Radiology } \\
\hline Abdominal CT scan & 145 & 5 & 725 & 7 & 1015 \\
\hline Thorax CT scan & 131 & 5 & 655 & 5 & 655 \\
\hline Pelvic MR scan & 339 & 4 & 1356 & 3 & 1017 \\
\hline Multidisciplinary meeting (primary) & 2647 & 6 & 15,882 & 10 & 26,470 \\
\hline Multidisciplinary meeting (repeated) & 1511 & 3 & 4533 & 3 & 4533 \\
\hline \multicolumn{6}{|l|}{ Treatment cost } \\
\hline Total cost & & & 235,211 & & 444,125 \\
\hline Treated women & & 64 & & 175 & \\
\hline Cost per treated woman & & & 3675 & & 2538 \\
\hline \multicolumn{6}{|l|}{ Follow-up after treatment } \\
\hline Midwife appointment & 98 & 34 & 3332 & 89 & 8722 \\
\hline Colposcopy appointment & 470 & 61 & 28,670 & 141 & 66,270 \\
\hline Biopsy histological analysis & 147 & 22 & 3234 & 90 & 13,230 \\
\hline Pap smear cytological analysis & 25 & 87 & 2175 & 226 & 5650 \\
\hline HPV test & 38 & 72 & 2736 & 191 & 7258 \\
\hline Total cost incl follow-up & & & 275,358 & & 545,255 \\
\hline Treated women & & 64 & & 175 & \\
\hline Cost per treated woman incl follow-up & & & 4302 & & 3116 \\
\hline
\end{tabular}

using a Markov model simulating the natural history of cervical cancer, plus empirical data to create a Swedish female cohort [34]. We concluded that self-sampling for HPV testing is cost-effective every 5 years among women aged over 35 years compared with cytologybased screening with Pap smears [34].

Swedish national guidelines on primary cervical screening with HPV testing and triage with liquid-based cytology among women aged over 30 years have recently been published $[35,36]$. The future societal costs are estimated to decrease as a result of fewer cancer cases needing healthcare, and the near-time healthcare costs of the screening program are estimated to increase as a result of more cases needing colposcopy, treatment and follow-up (https://www.socialstyrelsen.se/globalassets/ sharepoint-dokument/artikelkatalog/nationella-screeningprogram/2019-4-12.pdf). It is therefore of interest to assess alternative screening strategies that could both increase the participation in screening and reduce the overall costs and women suffering. This CEA on repeated self-sampling for HPV testing shows profitable results with respect to increasing participation at lower cost than conventional cytology in primary cervical screening.

\section{Conclusions}

The choice of a test, in addition to cost, is also highly influenced by the tests clinical performance and acceptance by women. Our earlier randomized study showed higher participation rate and the present study, based on 
the same population, shows reduced costs with homebased repeated self-sampling for HPV testing in comparison with clinic-based Pap smear sampling. Validated PCR-based HPV tests have shown good performance based on self-samples, but further studies are needed to evaluate the performance of different self-sampling kits, sample handling logistics, acceptance and costs, to guide policy-makers on the use of self-sampling for HPV testing in primary cervical screening.

\section{Abbreviations}

AIS: Adenocarcinoma in situ; ASCUS: Atypical squamous cells of unknown significance; CEA: Cost-effectiveness analysis; CIN1: Low-grade cervical intraepithelial neoplasia; CIN2 + : Cervical intraepithelial neoplasia grade 2 or more; HPV: Human papillomavirus; ICER: Incremental cost-effectiveness ratios; LEEP: Loop electrosurgical excision procedure; TZ3: Transformation zone 3

\section{Acknowledgements}

Not applicable.

\section{Authors' contributions}

All authors contributed the study plan. UG was responsible for the initial study and also in planning this study. MO was key person in planning and performing the study. RA performed nearly all colposcopies in the HPV selfsampling arm. RA noted all events in the retrieved database and manually checked in the patient files for treatment records. RA collected the actual costs of different interventions. EÖ performed the cost-effective analysis. IG was responsible for the database. RA and EÖ were major contributors in writing the manuscript. All authors took part in finalizing the manuscript and a final version was approved by all authors before submission.

\section{Funding}

The funders (UG: Cancerfonden: 190008 Pj 01 H, VR: 2015-02711 and Lions Cancer Research Foundation: 1050097), provided resources to plan the study, to collect and analyze the data and to write the report. The funders did not have any impact on the study design, choice of methods, collection of data, analyses or on the conclusions drawn. Open access funding provided by Uppsala University.

\section{Availability of data and materials}

All data generated or analyzed during this study are included in this published article and in a previously published article [18].

\section{Ethics approval and consent to participate}

The study was approved by the Regional Ethics Committee in Uppsala (Dnr 2012/099). Participants received written information and consent was given by opt-in, as approved by the Ethics Committee. Women were sent an invitation with a self-sampling kit and if they agreed to participate they performed self-sampling and sent the sample to the laboratory.

\section{Consent for publication}

Not applicable.

\section{Competing interests}

The authors declare that they have no competing interests.

\section{Author details}

'Department of Women's and Children's Health, Uppsala University, 75185 Uppsala, Sweden. ${ }^{2}$ Department of Women's and Children's Health, Karolinska Institutet, Tomtebodavägen 18A, 17177 Stockholm, Sweden. ${ }^{3}$ Department of Medical Epidemiology and Biostatistics, Karolinska Institutet, Nobels väg 12A, 17165 Stockholm, Sweden. ${ }^{4}$ Department of Immunology, Genetics, and Pathology, Biomedical Center, SciLifeLab Uppsala, Uppsala University, Box 815, 75108 Uppsala, Sweden.
Received: 13 May 2020 Accepted: 17 June 2020

Published online: 13 July 2020

\section{References}

1. Arbyn M. Trends of cervical cancer mortality in the member states of the European Union. Eur J Cancer. 1990;45(15):2640-8.

2. NORDCAN: Cancer Incidence, Mortality, Prevalence and Survival in the Nordic Countries, Version 8.2 (26.03.2019). Association of the Nordic Cancer Registries. Danish Cancer Society. Available from http://www.ancr.nu, Accessed 2 May 2019.

3. Walboomers JM, Jacobs MV, Manos MM, Bosch FX, Kummer JA, Shah KV, et al. Human papillomavirus is a necessary cause of invasive cervical cancer worldwide. J Pathol. 1999;189(1):12-9.

4. Naucler P, Ryd W, Tornberg S, Strand A, Wadell G, Elfgren K, et al. Human papillomavirus and Papanicolaou tests to screen for cervical cancer. N Engl J Med. 2007;357(16):1589-97.

5. Mayrand MH, Duarte-Franco E, Rodrigues I, Walter SD, Hanley J, Ferenczy A, et al. Human papillomavirus DNA versus Papanicolaou screening tests for cervical cancer. N Engl J Med. 2007;357(16):1579-88.

6. European guidelines for quality assurance in cervical cancer screening second edition. 2015. http://www.gisci.it/documenti/news/EW0115451ENN_ 002.pdf. Accessed 13 Jan 2020.

7. Ronco G, Dillner J, Elfström KM, Tunesi S, Snijders PJF, Arbyn M, et al. Efficacy of HPV-based screening for prevention of invasive cervical cancer: follow-up of four European randomised controlled trials. Lancet. 2014; 383(9916):524-32.

8. Mittal SBP, Muwonge R, Banerjee D, Ghosh I, Sengupta MM, Das P, Dey P, Mandal R, Panda C, Biswas J, Sankaranarayanan R. Risk of high-grade precancerous lesions and invasive cancers in high-risk HPV-positive women with normal cervix or CIN 1 at baseline-a population-based cohort study. Int J Cancer. 2017;140(8):1850-9.

9. Gyllensten U, Sanner K, Gustavsson I, Lindell M, Wikstrom I, Wilander E. Short-time repeat high-risk HPV testing by self-sampling for screening of cervical cancer. Br J Cancer. 2011;105(5):694-7.

10. Andrae B. Screening-preventable cervical cancer risks: evidence from a nationwide audit in Sweden. J Natl Cancer Inst. 2008;100(9):622-9.

11. Wikstrom I, Lindell M, Sanner K, Wilander E. Self-sampling and HPV testing or ordinary pap-smear in women not regularly attending screening: a randomised study. Br J Cancer. 2011;105(3):337-9.

12. Lindell M, Sanner K, Wikstrom I, Wilander E. Self-sampling of vaginal fluid and high-risk human papillomavirus testing in women aged 50 years or older not attending Papanicolaou smear screening. BJOG. 2012;1 19(2):245-8.

13. Darlin L, Borgfeldt C, Forslund O, Henic E, Hortlund M, Dillner J, et al. Comparison of use of vaginal HPV self-sampling and offering flexible appointments as strategies to reach long-term non-attending women in organized cervical screening. J Clin Virol. 2013;58(1):155-60.

14. Broberg G, Gyrd-Hansen D, Miao Jonasson J, Ryd ML, Holtenman M, Milsom I, et al. Increasing participation in cervical cancer screening: offering a HPV self-test to long-term non-attendees as part of RACOMIP, a Swedish randomized controlled trial. Int J Cancer. 2014;134(9):2223-30.

15. Verdoodt F, Jentschke M, Hillemanns P, Racey CS, Snijders PJ, Arbyn M. Reaching women who do not participate in the regular cervical cancer screening programme by offering self-sampling kits: a systematic review and meta-analysis of randomised trials. Eur J Cancer. 2015;51(16):2375-85.

16. Elfstrom KM, Sundstrom K, Andersson S, Bzhalava Z, Carlsten Thor A, Gzoul $Z$, et al. Increasing participation in cervical screening by targeting long-term nonattenders: randomized health services study. Int J Cancer. 2019;145(11): 3033-9.

17. Arbyn M, Verdoodt F, Snijders PJ, Verhoef VM, Suonio E, Dillner L, et al. Accuracy of human papillomavirus testing on self-collected versus cliniciancollected samples: a meta-analysis. Lancet Oncol. 2014;15(2):172-83.

18. Gustavsson I, Aarnio R, Berggrund M, Hedlund-Lindberg J, Strand AS, Sanner $\mathrm{K}$, et al. Randomised study shows that repeated self-sampling and HPV test has more than two-fold higher detection rate of women with CIN2+ histology than pap smear cytology. Br J Cancer. 2018;118(6):896-904.

19. Drummond M, Schulper M, Torrance GW. O'Brian BJ, Stoddart GL. Methods for the economic Evalutaion of Helath care Programmes. Edition $r$, editor. Oxford: Oxford university press; 2005

20. ScB. Consumer Price Index (CPI): Statistics Sweden; 2019. Available from: https://www.scb.se/en/finding-statistics/statistics-by-subject-area/prices-andconsumption/. [cited 2019 Mar 21]. 
21. Vijayaraghavan A, Efrusy MB, Mayrand MH, Santas CC, Goggin P. Costeffectiveness of high-risk human papillomavirus testing for cervical cancer screening in Quebec, Canada. Can J Public Health. 2010;101(3):220-5.

22. Berkhof J, Coupe VM, Bogaards JA, van Kemenade FJ, Helmerhorst TJ, Snijders PJ, et al. The health and economic effects of HPV DNA screening in the Netherlands. Int J Cancer. 2010;127(9):2147-58.

23. Lew JB, Simms KT, Smith MA, Hall M, Kang YJ, Xu XM, et al. Primary HPV testing versus cytology-based cervical screening in women in Australia vaccinated for HPV and unvaccinated: effectiveness and economic assessment for the National Cervical Screening Program. Lancet Public Health. 2017;2(2):e96-e107.

24. Pedersen K, Burger EA, Sy S, Kristiansen IS, Kim JJ. Cost-effective management of women with minor cervical lesions: revisiting the application of HPV DNA testing. Gynecol Oncol. 2016;143(2):326-33.

25. Haguenoer K, Sengchanh S, Gaudy-Graffin C, Boyard J, Fontenay R, Marret $H$, et al. Vaginal self-sampling is a cost-effective way to increase participation in a cervical cancer screening programme: a randomised trial. Br J Cancer. 2014;111(11):2187-96.

26. Burger EA, Sy S, Nygard M, Kim JJ. The cost-effectiveness of cervical selfsampling to improve routine cervical Cancer screening: the importance of respondent screening history and compliance. Cancer Epidemiol Biomark Prev. 2017:26(1):95-103.

27. Rozemeijer K, de Kok IM, Naber SK, van Kemenade FJ, Penning C, van Rosmalen J, et al. Offering self-sampling to non-attendees of organized primary HPV screening: when do harms outweigh the benefits? Cancer Epidemiol Biomark Prev. 2015;24(5):773-82.

28. Tsiachristas A, Gittins M, Kitchener H, Gray A. Cost-effectiveness of strategies to increase cervical screening uptake at first invitation (STRATEGIC). J Med Screen. 2018;25(2):99-109.

29. Vassilakos $\mathrm{P}$, Poncet $\mathrm{A}$, Catarino $\mathrm{R}$, Viviano $\mathrm{M}$, Petignat $\mathrm{P}$, Combescure $\mathrm{C}$. Cost-effectiveness evaluation of HPV self-testing offered to non-attendees in cervical cancer screening in Switzerland. Gynecol Oncol. 2019;153(1):92-9.

30. Asciutto KC, Henic E, Darlin L, Forslund O, Borgfeldt C. Follow up with HPV test and cytology as test of cure, 6 months after conization, is reliable. Acta Obstet Gynecol Scand. 2016;95(11):1251-7.

31. Rositch AF, Soeters HM, Offutt-Powell TN, Wheeler BS, Taylor SM, Smith JS. The incidence of human papillomavirus infection following treatment for cervical neoplasia: a systematic review. Gynecol Oncol. 2014;132(3):767-79.

32. Molloy M, Comer R, Rogers P, Dowling M, Meskell P, Asbury K, et al. High risk HPV testing following treatment for cervical intraepithelial neoplasia. Ir J Med Sci. 2016;185(4):895-900

33. Ostensson E, Alder S, Elfstrom KM, Sundstrom K, Zethraeus N, Arbyn M, et al. Barriers to and facilitators of compliance with clinic-based cervical cancer screening: population-based cohort study of women aged 23-60 years. PLoS One. 2015;10(5):e0128270.

34. Ostensson E, Hellstrom AC, Hellman K, Gustavsson I, Gyllensten U, Wilander $\mathrm{E}$, et al. Projected cost-effectiveness of repeat high-risk human papillomavirus testing using self-collected vaginal samples in the Swedish cervical cancer screening program. Acta Obstet Gynecol Scand. 2013;92(7): 830-40.

35. Strander Bea. Nationellt vårdprogram för prevention av livmoderhalscancer 2017 [updated 2018-12-11.

36. Screening för livmoderhalscancer - Rekommendation och bedömningsunderlag. 2015.

\section{Publisher's Note}

Springer Nature remains neutral with regard to jurisdictional claims in published maps and institutional affiliations.

\section{Ready to submit your research? Choose BMC and benefit from:}

- fast, convenient online submission

- thorough peer review by experienced researchers in your field

- rapid publication on acceptance

- support for research data, including large and complex data types

- gold Open Access which fosters wider collaboration and increased citations

- maximum visibility for your research: over $100 \mathrm{M}$ website views per year

At BMC, research is always in progress.

Learn more biomedcentral.com/submissions 